\title{
Single-particle states vs. collective modes: friends or enemies ?
}

\author{
T. Otsuka ${ }^{1,2,3,4,5}$, Y. Tsunoda ${ }^{3}$, T. Togashi ${ }^{3}$, N. Shimizu ${ }^{3}$, and T. Abe ${ }^{2}$ \\ ${ }^{1}$ RIKEN Nishina Center, 2-1 Hirosawa, Wako, Saitama 351-0198, Japan \\ ${ }^{2}$ Department of Physics, University of Tokyo, 7-3-1 Hongo, Bunkyo, Tokyo 113-0033, Japan \\ ${ }^{3}$ Center for Nuclear Study, University of Tokyo, 7-3-1 Hongo, Bunkyo, Tokyo 113-0033, Japan \\ ${ }^{4}$ Instituut voor Kern- en Stralingsfysica, K. U. Leuven, Celestijnenlaan 200D, B-3001 Leuven, Belgium \\ ${ }^{5}$ National Superconducting Cyclotron Laboratory,Michigan State University, East Lansing, Michigan 48824, USA
}

\begin{abstract}
The quantum self-organization is introduced as one of the major underlying mechanisms of the quantum many-body systems. In the case of atomic nuclei as an example, two types of the motion of nucleons, single-particle states and collective modes, dominate the structure of the nucleus. The collective mode arises as the balance between the effect of the mode-driving force (e.g., quadrupole force for the ellipsoidal deformation) and the resistance power against it. The single-particle energies are one of the sources to produce such resistance power: a coherent collective motion is more hindered by larger spacings between relevant single particle states. Thus, the single-particle state and the collective mode are "enemies" against each other. However, the nuclear forces are rich enough so as to enhance relevant collective mode by reducing the resistance power by changing single-particle energies for each eigenstate through monopole interactions. This will be verified with the concrete example taken from $\mathrm{Zr}$ isotopes. Thus, the quantum self-organization occurs: single-particle energies can be self-organized by (i) two quantum liquids, e.g., protons and neutrons, (ii) monopole interaction (to control resistance). In other words, atomic nuclei are not necessarily like simple rigid vases containing almost free nucleons, in contrast to the naïve Fermi liquid picture. Type II shell evolution is considered to be a simple visible case involving excitations across a (sub)magic gap. The quantum self-organization becomes more important in heavier nuclei where the number of active orbits and the number of active nucleons are larger.
\end{abstract}

\section{Introduction}

The underlying mechanisms of the many-body structure of atomic nuclei have been studied over decades as one of the most important objectives of nuclear physics. It has then been understood usually that there are two types of dominant motion of nucleons in the atomic nucleus : single-particle states and collective modes. Regarding the single-particle states, Mayer and Jensen introduced the shell structure and associated magic numbers [1-3]. The nuclear shell model developed on these concepts has been extremely successful in the description of the structure of many nuclei (see for example [4-6]). The collective modes include various cases. Among them, the deformation of the nuclear shape has been studied since Rainwater [7], and Bohr and Mottelson [8, 9]. The nuclear shapes have been one of the major focuses of the nuclear structure physics, including spherical, vibrational and rotational ones [10]. The relation between the singleparticle states and the collective modes has naturally become of much interest, as described by Bohr and Mottelson in [10] as "the problem of reconciling the simultaneous occurrence of single-particle and collective degrees of freedom and exploring the variety of phenomena that arise from their interplay".

The atomic nucleus is a many-body quantum system comprised of protons and neutrons, which is often consid- ered to be described in terms of Landau's Fermi Liquid picture. In a somewhat simplified expression of this picture, protons and neutrons of a nucleus are in a mean potential which is like a rigid "vase", and those nucleons are like free particles moving in this vase, interacting weakly among themselves through a "residual interaction". The single-particle energies (SPE) of such a system exhibit the shell structure, and are split in general. If the splitting is large enough, the many-body structure is dominated by the SPEs: nucleons occupy the lowest single particle states in the ground state, the next lowest configuration gives us the first excited state, and so forth. In such cases, the correlations due to the interaction between nucleons may contribute, but their effects are minor, more or less, compared to the effects of SPE splittings. However, if the energy gain from such correlations overcomes the relevant SPE splittings, a collective mode dominates the structure of the ground and low-lying states. Although the understanding of the relation between the single-particle states and the collective modes has been pursued in many ways, it seems to remain an open problem. For instance, G.E. Brown had kept, throughout his life, the question, "how single particle states can coexist with collective modes" as quoted from "Fermi liquid theory: A brief survey in memory of Gerald E. Brown" in [11]. We shall present a novel mechanism which is closely related to this problem. 

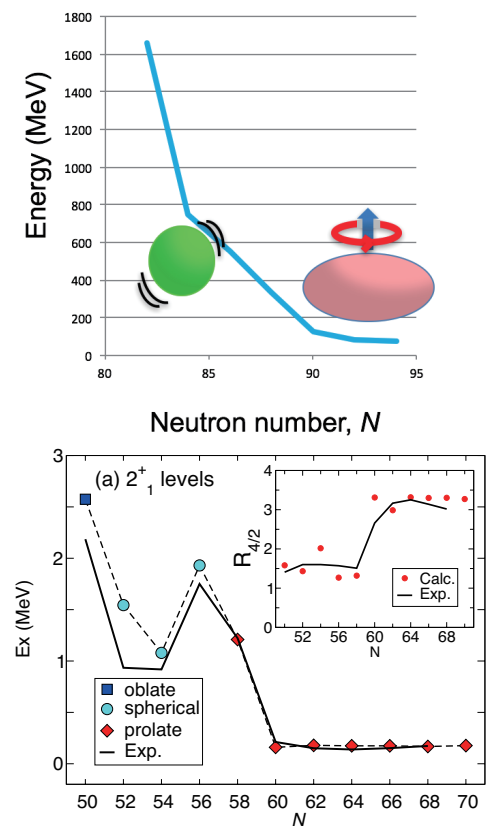

Figure 1. Systemtic changes of the $2_{1}^{+}$level in (upper) Sm and (lower) $\mathrm{Zr}$ isotopes, as functions of $N$. Data taken from [14] for $\mathrm{Sm}$. The right panel is based on [12].

\section{Nuclear shapes and quantum phase transition}

We shall focus on the quadrupole deformation of the nuclear shape in this talk, while the scope can be extended. Figure 1 exhibits the excitation energy of $2_{1}^{+}$state, or the $2_{1}^{+}$level, for $\mathrm{Sm}$ and $\mathrm{Zr}$ isotopes as a function of the neutron number, $N$. In the Sm chain, the $2^{+}$level comes down rather gradually, similarly to many other isotopic chains. As shown in Fig. 1, a higher $2^{+}$level corresponds to a spherical shape and its surface oscillation, while a lower $2^{+}$level implies an ellipsoidal deformed shape and the rotation of the ellipsoid. On the other hand, in the $\mathrm{Zr}$ chain, the $2^{+}$level drops down abruptly in moving from $N=58$ to 60 . Due to this abrupt change, this phenomenon can be referred to as a quantum phase transition [12]. Likewise, the ground-state structure of the $\mathrm{Zr}$ isotopes is changed drastically between $N=58$ and 60 , also from the sphere to the strongly deformed ellipsoid. The Monte Carlo Shell Model (MCSM) describes both situations including the abrupt change with the same Hamiltonian $[12,13]$.

\section{Monte Carlo Shell Model}

We here sketch the MCSM briefly $[15,16]$. The MCSM is a recent method to obtain eigensolutions of the shellmodel calculation in nuclear physics. The shell-model calculation is quite similar to the configuration interaction (CI) calculation in other fields of science. The major differences are that (i) the two ingredients, protons and neutrons, are taken instead of electrons, and (ii) nuclear forces are considered instead of Coulomb or other forces. In conventional shell-model calculations, the matrix of the Hamiltonian with respect to many Slater determinants is diagonalized. Because a huge number of configurations are needed for the description of large systems, the dimension of the matrix can be enormous, making the calculation infeasible. On the other hand, many interesting and important problems lie beyond this limit. The MCSM provides a breakthrough in this regard. The MCSM is very different from the conventional shell-model calculation. A set of Slater determinants, called MCSM basis vectors, is introduced, and the diagonalization is performed in the Hilbert space spanned by the MCSM basis vectors. Each MCSM basis vector is a Slater determinant composed of single-particle states that are superpositions of the original single-particle states, and the amplitudes of these superpositions are determined through a combination of stochastic and variational methods. Even when the dimensions are in the order of $10^{23}$ for the conventional shell model, the problem can be solved, to a good approximation, with up to approximately $100 \mathrm{MCSM}$ basis vectors.

\section{Quantum Phase Transition in Zr isotopes}

We shall look into the structure changes in $\mathrm{Zr}$ isotopes based on the MCSM calculation. The upper panel of Fig. 2 shows the occupation numbers of proton orbits for some states. The $\mathrm{g}_{9 / 2}$ orbit is almost empty in the $0_{1}^{+}$state of ${ }^{98} \mathrm{Zr}$, whereas it is occupied by about 3.5 protons in its $0_{2}^{+}$ state. Note that this $0_{1}^{+}\left(0_{2}^{+}\right)$state is spherical (deformed). Such changes, including the numbers of proton holes in the $p f$ orbits, result in substantial shifts of the neutron (effective) SPEs as shown schematically in the middle panel of Fig. 2. The proton-neutron monopole interaction (wavy line in the figure) generates those shifts. The right panel depicts the actual neutron effective SPEs. One notices a substantial change in SPE for the different states considered. One sees that the spacing between the $d_{5 / 2}$ and $g_{7 / 2}$ orbits is nearly $5 \mathrm{MeV}$ for the $0_{1}^{+}$state of ${ }^{98} \mathrm{Zr}$, but it is reduced to about $2 \mathrm{MeV}$ in $0_{2}^{+}$state. Such a reduced splitting is found also in the $0_{1}^{+}$state of ${ }^{100} \mathrm{Zr}$ which is also strongly deformed.

We now discuss why the SPEs are so different between spherical and deformed states. We first point out that the nuclear deformation at low excitation energy is a JahnTeller effect [17], meaning that the collective motion causing the deformation occurs as a consequence of coherent contributions from some relevant orbits near the Fermi energy. For such coherent effects, larger splittings of SPEs weaken the coherence, leading to less collectivity. On the other hand, the monopole interaction can change the effective SPEs depending on the occupancy of the other nucleons. If the monopole interaction were uniform, no configuration dependence would appear, and this change should 

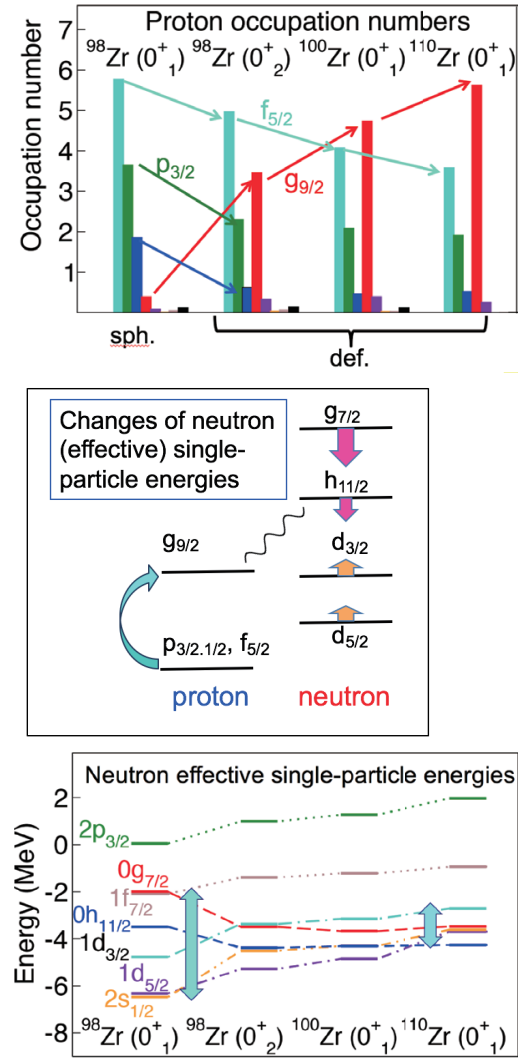

Figure 2. (upper) Occupation numbers of proton orbits of $\mathrm{Zr}$ isotopes. (middle) Schematic illustration of the changes of neutron (effective) single-particle energies in $\mathrm{Zr}$ isotopes. (lower) Actual values of neutron (effective) single-particle energies obtained in the calculation of [12]. Upper and lower panels are based on [12]

be absent. The tensor-force component of the nuclear force makes the monopole interaction attractive or repulsive, depending on the combination of the orbits [18, 19]. This is certainly against the uniformity, and its effect can be crucial. The central-force component changes its magnitude also depending on the combination of the orbits mainly due to varying overlaps of radial wave functions of single-particle states [19]. Thus, the monopole interaction is indeed far from being uniform, and the selection of favored configurations can move the SPEs of relevant orbits substantially. If relevant SPEs can be made closer to being degenerate, it helps the deformation. We shall formulate this novel mechanism in the next section.

\section{Quantum self-organization}

The nuclear deformation is determined by the balance between the effect of the collective-mode driving force and the resistance power against this collective mode. A schematic expression of this property is,

$$
\text { deformation }=\frac{\text { quadrupole force }}{\text { resistance power }} .
$$

The collective-mode driving force is the quadrupole (or quadrupole-quadrupole) interaction in the case of the ellipsoidal shape. This interaction is one of the major components of the proton-neutron realistic force. A typical example of the resistance power is the pairing interaction, which tends to make the shape more spherical because all time-reversal pairs are equally favored. Keeping the pairing interaction aside, we shall consider another source of the resistance power. That is the SPEs.

We here propose a novel mechanism called, Quantum Self-Organization. This mechanism means the following property: Atomic nuclei can "organize" their single-particle energies by taking particular configurations of protons and neutrons, optimized for each eigenstate, thanks to orbit-dependences of monopole components of nuclear forces (e.g., tensor and central forces). This results in an enhancement of Jahn-Teller effect, i.e., an enhancement of the collective mode. The deformation andk quantum self-organization can be linked in a non-linear way with a positive feedback : once some nucleons are excited to particular orbits, the SPEs are shifted in favor of a larger deformation. A larger deformation can promote such excitations with more nucleons. This cycle continues until a self-consistency is achieved, whereas intermediate situations are skipped. In many cases, massive excitations are involved, and the particle-hole hierarchy is broken, for instance, a $6 \mathrm{p}-6 \mathrm{~h}$ deformed state comes right after a $2 \mathrm{p}-2 \mathrm{~h}$ near-spherical state, skipping 4p-4h state [20].

The property shown in eq. (1) is somewhat analogous to the relation,

$$
\text { electric current }=\frac{\text { voltage }}{\text { resistance }},
$$

where the electric current, voltage and resistance mean the usual quantities regarding the electricity. The higher voltage produces a higher current, but the current can be increased also by decreasing the resistance. The quantum self-organization implies that the atomic nucleus finds particular configurations which decrease the resistance power.

The most favorable configurations and associated SPEs vary for individual eigenstate even within the same type of the collective mode. For instance, prolate, oblate or triaxial shapes belong to the quadrupole deformation, but can appear with different patterns of the SPEs within the same nucleus. The oblate shape is less affected by the quantum self-organization, because smaller numbers of nucleons on unique-parity orbits are the key element of the oblate shape in most cases. In those cases, the organization of many orbits are rather irrelevant, and the quantum self-organization may not occur to a sizable extent. This feature has been verified with concrete cases. On the other hand, many orbits contribute coherently to the prolate deformation, and the quantum self-organization can produce crucial effects. This has been confirmed by changing the 


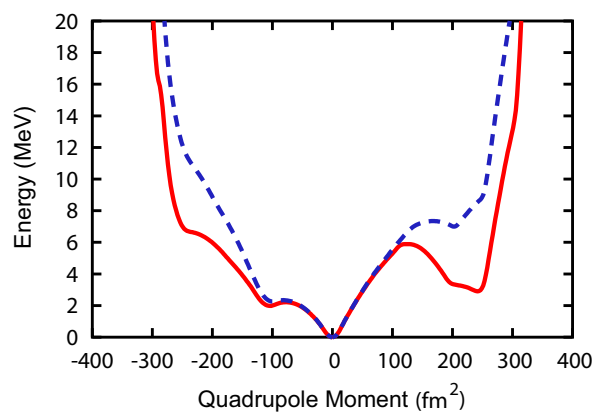

Figure 3. Potential Energy Surface with the axially symmetric deformation for ${ }^{68} \mathrm{Ni}$. The red solid line denotes the energy of the constrained Hartree-Fock calculation with the original Hamiltonian. The blue dashed line implies the same calculation except that the quantum self-organization is suppressed (see the text). Figure taken from [20].

monopole interactions, for instance, closer to the uniform one.

We present a concrete example by taking the case of the prolate band in ${ }^{68} \mathrm{Ni}[20]$. The monopole interaction between the neutron $1 g_{9 / 2}$ orbit and the proton $1 f_{5 / 2}$ orbit is more attractive than that between the neutron $1 g_{9 / 2}$ orbit and the proton $1 f_{7 / 2}$ orbit mainly due to the robust property of the monopole interaction of the tensor force, and this difference serves as the major origin of the quantum self-organization in this particular case: more neutrons in the $1 \mathrm{~g}_{9 / 2}$ orbit reduces the $1 \mathrm{f}_{7 / 2}-1 \mathrm{f}_{5 / 2}$ spinorbit splitting for protons $[18,19,21]$. The effect of this difference on the deformation can be seen quantitatively by replacing the strengths of these monopole interactions with the average of their original values, i.e., the same value. Likewise, we reset the monopole interaction between the neutron $1 f_{5 / 2}$ and the proton $1 f_{7 / 2}$ orbits and that between the neutron $1 f_{5 / 2}$ and the proton $1 f_{5 / 2}$ orbits. These modifications correspond basically to the removal of the tensor-force monopole contributions, and are nothing but the suppression of the present effects of the quantum self-organization. The resulting Potential Energy Surface is shown in Fig. 3 for the axially symmetric deformation compared with that obtained from the original Hamiltonian. Around the spherical minimum the energy curves of the two calculations are similar, however, when going to stronger deformation values, the two approaches differ substantially. In particular, the prolate profound local minimum, seen in the original calculation (red solid line), is pushed up by about $4 \mathrm{MeV}$, if the quantum self-organization is suppressed as described above (blue dashed line). Thus, the quantum self-organization is a part of the crucial mechanisms producing the nuclear deformation.

At this point, we mention that the SPE being discussed corresponds somehow to the spherical terms in the Nilsson model [10] which are comprised of the $\ell \ell$ and $\ell s$ terms as well as the harmonic-oscillator-quanta term. As their strengths are independent of the deformation, the present effect is not included in the Nilsson model.

Type-II shell evolution [20] has been discussed, for instance, in $\mathrm{Co} / \mathrm{Ni}$ region [21-23], where neutrons are excited from the $p f$ shell to $g_{9 / 2}$ across the $N=40$ sub-magic gap. The neutrons in $g_{9 / 2}$ and neutron holes in $f_{5 / 2}$ provide similar sizable monopole effects, as discussed above. A smaller $1 f_{7 / 2}-1 f_{5 / 2}$ spin-orbit splitting for protons reduces the resistance power against deformation, pulling down the prolate band as seen in Fig. 3. Type II shell evolution was introduced as the particle-hole excitation over a magic or sub-magic gap. Clearly, this kind of mechanism is a very simple and visible case of the quantum self-organization. On the other hand, the quantum selforganization can occur certainly in more complex ways. Such a complex way may be found in the shape transition of Sm isotopes (see Fig. 1), where no magic or sub-magic gap is involved. We can see the spherical-vibrationalrotational shape evolution in MCSM calculations, as will be reported in detail elsewhere.

Likewise, the shape coexistence in $\mathrm{Hg} / \mathrm{Pb}$ isotopes have been studied. In those cases, the quantum selforganization gives intriguing contributions on the pattern of the shape coexistence, as reported also elsewhere.

\section{Summary and Perspectives}

We presented a novel mechanism on the relation between single-particle states and collective modes. A summary is given below.

- The atomic nuclei are not like simple rigid vases containing almost free nucleons interacting only weakly. This is in contrast to the naïve Fermi liquid picture.

- Nuclear forces are rich enough to change single-particle energies for each eigenstate, and can lead to the quantum self-organization.

- Single-particle energies can be self-organized, being enhanced by

(i) two quantum liquids (e.g., protons and neutrons)

(ii) two major force components

e.g., quadrupole interaction: to drive collective mode monopole interaction: to control resistance

- Type II shell evolution is a simple visible case involving excitations across (sub)magic gap.

- Actual cases such as shape coexistence, quantum phase transition, octupole vibration/deformation, super deformation, etc. can be studied with this scope.

- The quantum self-organization becomes more important in heavier nuclei where the number of active orbits and the number of active nucleons are larger. With larger numbers of them, the effects of the organization can be more significant. This feature may be linked to fission and superheavy elements. On the other hand, the quantum self-organization may not be so visible in light nuclei except for particular cases like intruder bands or cluster (or multiple particle-hole excited) states. 
- Time-dependent version of quantum self-organization may be of another interest for reactions and fission.

The MCSM calculations were performed on the K computer at RIKEN AICS (hp140210, hp150224, hp160211,hp170230). This work was also supported in part by the HPCI Strategic Program (The origin of matter and the universe) and "Priority Issue on Post-K computer" (Elucidation of the Fundamental Laws and Evolution of the Universe) from MEXT and JICFuS.

\section{References}

[1] M. G. Mayer, Phys. Rev., 75, 1969 (1949).

[2] O. Haxel,J. H. D. Jensen and H. E. Suess, Phys. Rev., 75, 1766 (1949).

[3] M. G. Mayer and J. H. D. Jensen, Elementary Theory of Nuclear Shell Structure (Wiley, New York, 1955).

[4] de Shalit A. and I. Talmi, Nuclear Shell Theory (Dover,2004).

[5] K. L. G. Heyde, The Nuclear Shell Model (Springer,1990).

[6] E. Caurier, G. Martinez-Pinedo, F. Nowacki, A. Poves and A. P. Zuker, Rev. Mod. Phys., 77, 427 (2005).

[7] J. Rainwater, Phys. Rev., 79, 432 (1950).

[8] A. Bohr, Mat. Fys. Medd. Dan. Vid. Selsk., 26 (1952).

[9] A. Bohr and B. R. Mottelson, Mat. Fys. Medd. Dan. Vid. Selsk., 27 (1952).

[10] A. Bohr and B. R. Mottelson, Nuclear Structure, vol. 2 (Benjamin, New York, 1975).
[11] T. Schaefer, Nucl. Phys. A, 928, 180 (2014).

[12] T. Togashi, Y. Tsunoda, T. Otsuka, and N. Shimizu, Phys. Rev. Lett., 117, 172502 (2016).

[13] C. Kremer, S. Aslanidou, S. Bassauer, M. Hilcker, A. Krugmann, P. von Neumann-Cosel, T. Otsuka,N. Pietralla, V. Y. Ponomarev, N. Shimizu, et al., Phys. Rev. Lett., 117, 172503 (2016).

[14] NNDC, Nudat 2, http://www.nndc.bnl.gov/nudat2/ (2016).

[15] T. Otsuka et al., Prog. Part. Nucl. Phys., 47, 319 (2001).

[16] N. Shimizu et al., Prog. Theor. Exp. Phys., 2012, 01A205 (2012).

[17] H. A. Jahn and E. Teller, Proc. R. Soc. A, 161, 220 (1937).

[18] T. Otsuka, T. Suzuki, R. Fujimoto, H. Grawe, and Y. Akaishi, Phys. Rev. Lett., 95, 232502 (2005).

[19] T. Otsuka, T. Suzuki, M. Honma, Y. Utsuno, N. Tsun-oda, K. Tsukiyama, and M. Hjorth-Jensen, Phys. Rev. Lett., 104, 012501 (2010).

[20] T. Otsuka and Y. Tsunoda, J. Phys. G, 43, 024009 (2016).

[21] Y. Tsunoda, T. Otsuka, N. Shimizu, M. Honma, and Y. Utsuno, Phys. Rev. C, 89, 031301(R) (2014).

[22] A. Morales, G. Benzoni, et al., Phys. Lett. B, 765, 328 (2017).

[23] S. Leoni, B. Fornal, et al., Phys. Rev. Lett., 118, 162502 (2017). 